\title{
Hamlet: Force/Position Controlled Hexapod Walker - Design and Systems
}

\author{
Michael R. Fielding*‡ ${ }^{*}$, Christopher J. Damaren ${ }^{\dagger}$ and Reg Dunlop*
}

\begin{abstract}
Hamlet is a hexapod walker constructed at the University of Canterbury, New Zealand, to test the effectiveness of combined force and position control to achieve robust, adaptable walking over rough and unknown environments. In particular the authors propose that compliance behaviours using the horizontal force measured at the feet will allow the robot to navigate slippery and dynamic surfaces such as gravel and rocks that move under contact with the feet. The robot and its systems are described, and the position-based compliance control strategy used is elaborated on.
\end{abstract}

Keywords - position-based compliance control, walking hexapod robot, gait generation

\section{INTRODUCTION}

Walking machines are desirable because they can navigate terrain features that are similar in size to the size of the robot, whereas wheeled and tracked vehicles are only suitable for obstacles smaller that half the diameter of the wheel. Furthermore, if given an ability to find locally horizontal footholds in regionally steep terrain, they can climb surprising angles. Applications potentially include (on earth and beyond) mining, exploration, military, rescue, industrial environments and recreation.

Since the first steps of the OSU Hexapod [1], the Adaptive Suspension Vehicle [2] and Genghis [3], hexapod walking robots have received considerable academic and even public attention. But despite the application of complex models of the robot dynamics (for example [4], [5], [6]) and optimisation techniques (for example [7], [8], [9], [10]), the huge increase in available computational power since the eighties has not yielded such a significant improvement in the robot behaviour.

It may be said that walking over a rough and unknown environment is a complex behaviour, requiring a complex system. But a simple system (the robot) interacting with a complex one (the environment) can produce complex behaviour. This project aims to take a different path to much of the current research by returning to a focus on simple behaviours. This is sometimes called biologicallyinspired robotics, as the behaviours and implementation are designed to mimic our understanding of how simple biological systems, such as insects, work. Such robots are often implemented in biologically motivated ways too - neural networks, distributed processing and biomorphic design for example [3], [10]. This project shows that the behaviour of robots with traditional functional design and linear soft-

* Dept. of Mechanical Engineering, University of Canterbury, New Zealand

${ }^{\dagger}$ Institute for Aerospace Studies, University of Toronto, Canada

${ }^{\ddagger}$ Corresponding author, m.fielding@ieee.org, fax +64 33642078 ware can still be inspired by observations from biological systems.

The basic tenet of the robot Hamlet is that by using the forces at the feet to modify their positions during stance, on a leg by leg basis rather than globally, dependable and effective walking can be achieved over a rough environment. This should be able to be achieved without a priori knowledge of the landscape or its properties, ie: without vision or mapping systems.

Compliance behaviour in the vertical direction has long been demonstrated to improve robot walking over uneven surfaces [1]. In particular it reduces the variation in body height and orientation when traversing obstacles.

Recent biological evidence supports the use of horizontal force-control in biologically-inspired hexapod walkers. Bartling [11] reports that Carausius morosus exerted a brief force directed anteriorly ${ }^{1}$ and proximally ${ }^{2}$ at the beginning of the stance. Bartling suggests that the insect detects the stiffness of the ground immediately following footfall, during the anterior directed force. No biological mechanism is known for continuously measuring the ground stiffness.

Very compliant motion in the horizontal directions may allow the robot to work on very non-linear surfaces that slip (such as sand or pebbles) or roll (such as rocks) when a robot walks over them. Under strict position control the footing would be lost and the robot would stumble or fall, requiring exception handling behaviours in order to recover. Compliant control in the horizontal direction is intended make recovery from these situations an emergent property, obviating the need for specialised behaviours. This would have advantages of simplicity and robustness - the robot would not require specialised behaviours, and it would be more stable if it could avoid having to reswing the leg, instead moving it to make the most of the current foothold. In fact, the robot would probably still require exception handling for very difficult situations, and for avoiding obstacles in the swing phase.

Compliance behaviour also deals neatly with the kinematic redundancy inherent in hexapod walking machines, regardless of the number of stance legs, because the legs are still under some position control. Foot interaction forces caused by the compliance will actually reduce the loading on the motors, reducing power consumption.

Unlike many researchers, the intention is not to adapt an ideal or prescribed gait to a rough terrain, but rather begin from the standpoint that flat terrain is just a special case of a natural terrain, as in Celaya and Porta [12].

\footnotetext{
${ }^{1}$ Towards the front.

${ }^{2}$ Towards the body.
} 
Therefore control algorithms need not change depending on an interpretation of the terrain, although this does not preclude the behaviour from changing as the environment provides unexpected inputs to the system.

On rough ground, insects such as locusts [13] and stick insects [14] walk with a free gait (a patternless, nonrepeating gait), while on smoother ground they adopt a periodic gait. The anterior extreme position ${ }^{3}$ (AEP) of a leg can be strongly influenced by the posterior extreme position $^{4}$ (PEP) of its anterior neighbour as exhibited in the stick insect Carausius morosus [15], [16]. This places the tarsus of the middle or hind legs close to the tarsus of the their respective anterior neighbour, increasing the likelihood of finding a good foothold. This behaviour was not displayed by locusts [13], which may be attributed to the different habitats of the animals.

Cruse et al. [17] and Weidemann et al. [10] have shown that careful addition of similar influences between the legs of a hexapod robot can yield a robust free gait that maintains stability and desired movement, and yet also tends toward a tripod or metachronal wave gait on flat ground. More specifically, legs are prevented from lifting or triggered to lift by their posterior ${ }^{5}$ neighbours, and no overall checking is necessary to ensure that the robot does not stumble.

One clear advantage of this 'soft' distributed control technique, as compared to 'hard' centralised control [18], [19], [20], is that the effect on the overall gait of interruptions to one leg's movements are managed without resorting to specialised gait behaviours. In most cases the robot can continue to walk using the remaining legs while the impeded leg executes a short re-swing to clear the obstacle. Weidemann et al. [10] showed that completely disabling a leg was not enough to stop the robot (a simulation in this case) from walking - the system ensured that it didn't stumble by preventing other legs from lifting, and over a few steps it recovered the original pattern. With a central pattern generator $(\mathrm{CPG})$ the gait must be stopped while the exception is handled, halting the motion of the robot abruptly. Naturally even a soft algorithm may need to stop walking in the case of a major, or impassable, obstruction or incapacitation.

Switching smoothly between gaits is also difficult with CPG algorithms, whereas the soft algorithms display smooth gait transition as an emergent property.

Goal-oriented path planning and navigation are the subject of much research for both legged and wheeled robots, for example [19]. This project does not address this issue, instead leaving the navigation of the robot under direct operator control.

The rest of this paper describes the mechanical, electronic and software design of a small hexapod, Hamlet, and the implementation of a force-position control scheme

\footnotetext{
${ }^{3}$ The end of the swing phase of leg movement when the foot is placed on the ground.

${ }^{4}$ The end of the stance phase of leg movement when the foot lifts off the ground.

${ }^{5}$ Immediately behind.
}

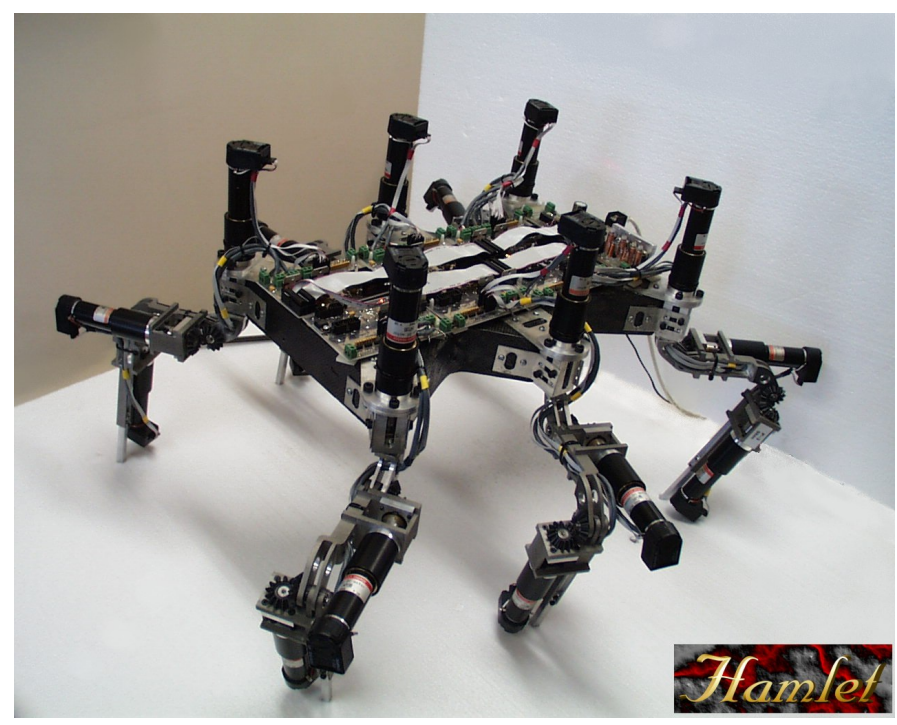

Fig. 1

HAMLET STANDing ON A TABle.

for the feet.

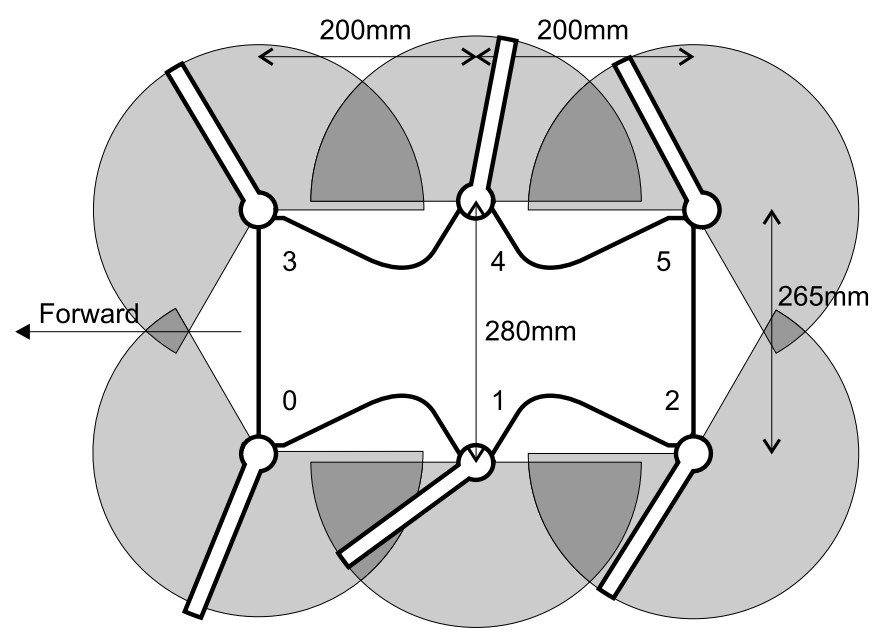

Fig. 2

The LOCATIONS OF THE $\alpha$ JOINTS AND THE NUMBERING OF THE LEGS. GREY SECTORS ARE THE APPROXIMATE ARCS INSCRIBED BY THE END OF THE FEMORA (ACTUALLY THE MOTOR SINCE IT PROTRUDES PAST THE FEMUR-TIBIA JOINT) WHEN THE FEMUR IS PARALLEL WITH THE CHASSIS PLANE.

\section{Mechanical}

Fig. 1 shows the general layout of the robot. The robot weighs about $13 \mathrm{~kg}$ and stands $400 \mathrm{~mm}$ high in this photo. Fig. 2 shows the locations of the six identical legs and the approximate movements available about the $\alpha$ axis (directly out of the page) of each leg before the leg hits the chassis. The high mobility of the $\alpha$ axis is intended to allow the legs to explore the environment when searching for a foothold and provides more scope for manoeuvring. The 


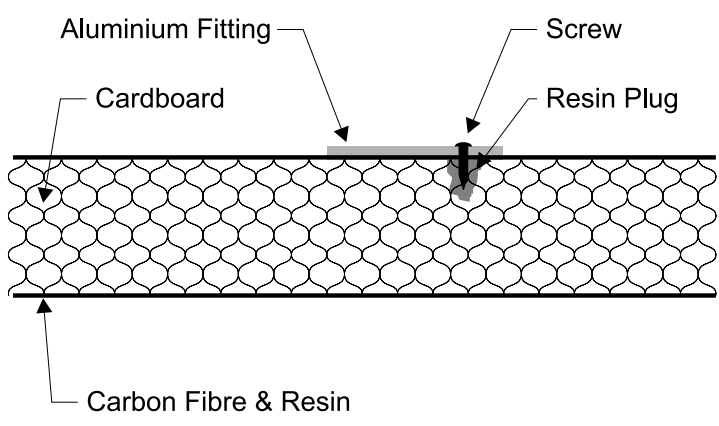

Fig. 3

A CROSS-SECtion OF the COMPOSite CHASSIS SHOWing HOW THE LEG FITTINGS ARE ATTACHED.

leg attachments all lie in the same plane, with all the $\alpha$ axes parallel.

The chassis (Fig. 3) is a sandwich of carbon fibre over corrugated cardboard which was found to be extremely stiff and light. Fittings for the legs are sheet aluminium attached by resin and stainless steel screws into injected resin plugs.

The legs (Fig. 4) are all identical, machined from aluminium and have three revolute joints each. The first two (coxa-trochanter denoted by $\alpha$ and trochanter-femur denoted by $\beta$ ) are orthogonal to each other, and the third (femur-tibia denoted by $\gamma$ ) is parallel with the second.

All the joints use identical 10W DC motors running through 1:246 planetary reduction gearboxes providing $4.5 \mathrm{Nm}$ torque in both directions. On the $\beta$ and $\gamma$ axes they are coupled through Oldham Couplings and nylon bevel gears to align them with the joint axes; on the $\alpha$ axes only an Oldham Coupling is used. The meshing of the bevel gears is adjustable to reduce backlash.

Optical quadrature encoders provide angle measurements. Calibration of their offset is achieved by gently moving the joints until they hit the chassis, which is a known datum for each joint provided a certain configuration and order is used. Calibration can be carried out without operator intervention.

The end of the tibia is provided with a three axis load cell using strain gauges, shown in Fig. 5. Note that the load cells are not fitted in Fig. 1. The design was undertaken using Finite Element Modelling. Amplifiers are located on the load cell for improved noise performance. Careful application of the strain gauges has minimised drift, although it is possible to recalibrate when the leg is in the air if necessary. A ball-jointed foot ensures that no torques are transmitted to the load cell that would upset the force readings, and also provides a large surface area for grip and to prevent sinking into soft environments. The minimal mass located on the foot means that the sensor dynamics can be ignored.

\section{Electronics And Software}

Hamlet has little processing and no battery power onboard, as autonomy is not necessary to achieve the project

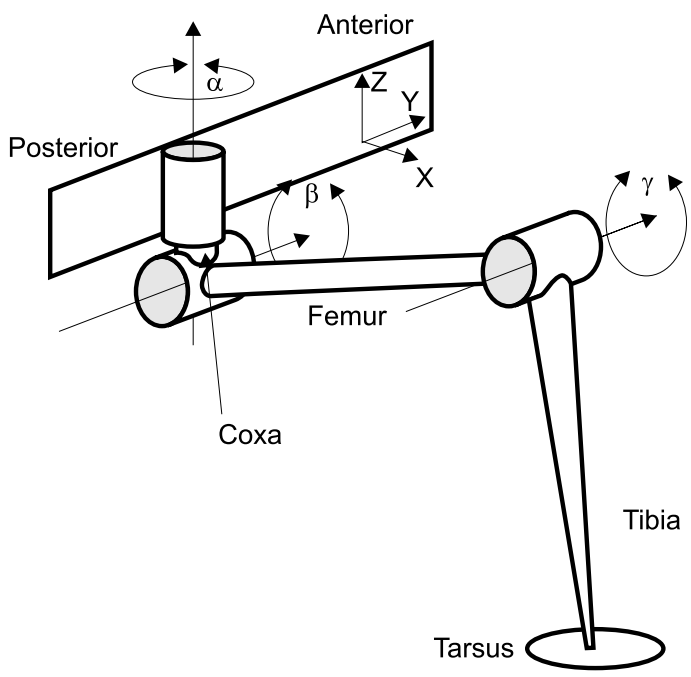

Fig. 4

The aXes AND PARTS OF THE LEG, With the Chassis COORDinate SYSTEM.

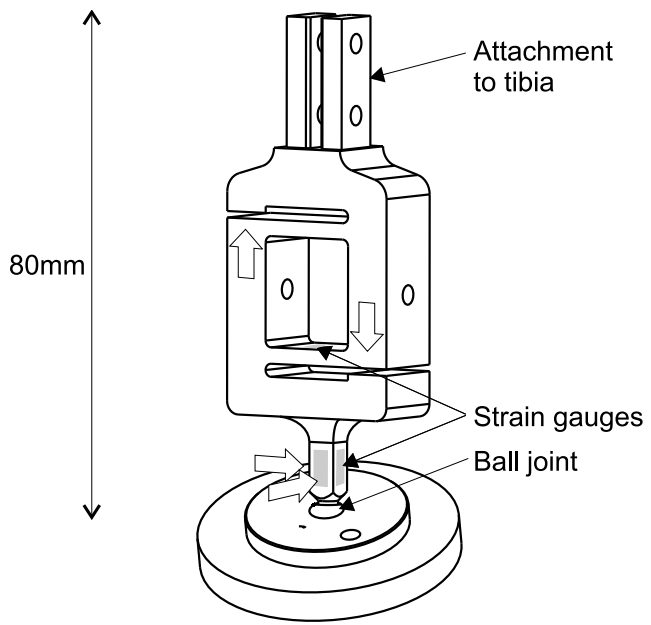

Fig. 5

A Diagram of The Force SENSOR on EACH FOOT. THE FORCE ON THE FOOT IN THE VERTICAL DIRECTION IS MEASURED BY A FULL BRIDGE ON THE THIN HORIZONTAL BEAMS, WHILE THE HORIZONTAL FORCES ARE MEASURED BY HALF BRIDGES NEAR THE BALL JOINT.

goals. A full duplex 4Mbit/s serial link using RS-485 over a twisted pair channel provides a data link between the DSPs and the robot (Fig. 6), transferring data at the sample rate of $200 \mathrm{~Hz} .24$ bit words were chosen because of the resolution of the angle encoders. Sign extension to 32 bits is done in hardware at both ends.

Power is delivered by a single-ended $18 \mathrm{~V}$ DC supply, converted to a variety of voltages on the robot by a switching power supply. Total current draw when standing is approximately $2 \mathrm{~A}$. The power supply module also supports the RS-485 interface to the serial link and is designed to accommodate a future upgrade to wireless operation.

The electronics on the robot are modularised with each 


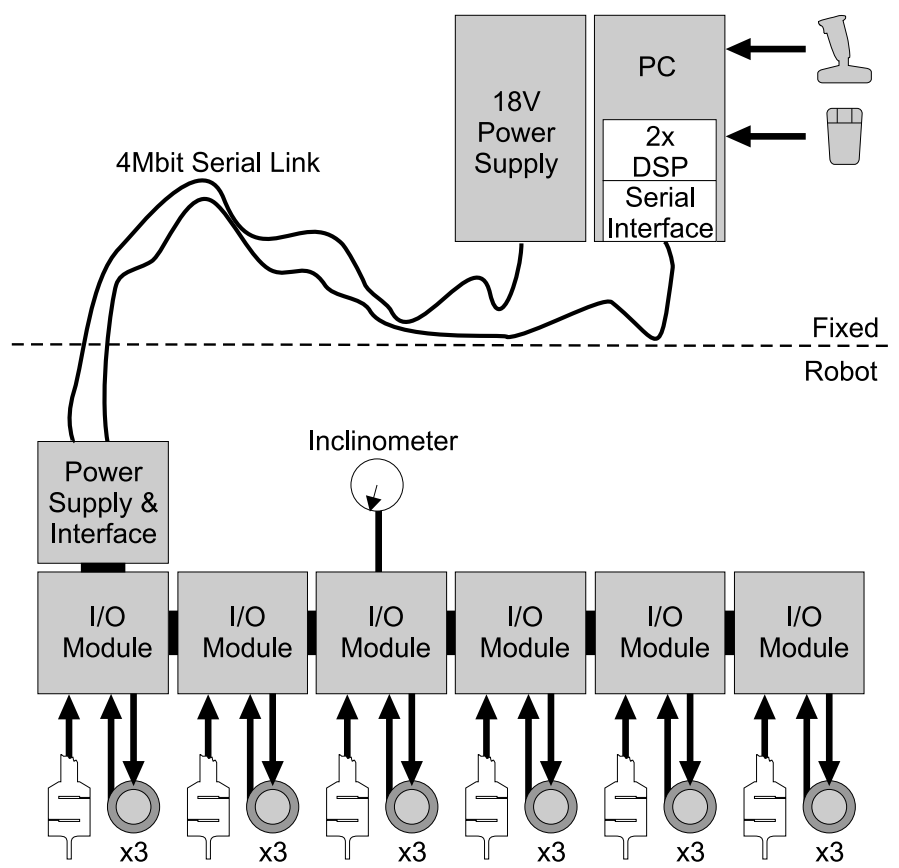

Fig. 6

The Processing is DONE IN The PC AND DSPs, With DATA SENT TO AND FROM THE ROBOT VIA A HIGH SPEED SERIAL LINK. $6 \mathrm{I} / \mathrm{O}$ MODULES ARE DAISY CHAINED TOGETHER ON THE ROBOT, EACH PROVIDING FUNCTIONS FOR ONE LEG.

module based on a Xilinx Field-Programmable Gate Array. There is one module for each leg, with a 14 bit 8-channel A/D, 12 bit PWM outputs, 24 bit encoder angle and velocity measurement (by measuring the time interval between pulses) and some digital I/O. Each module has its own analogue power supplies to minimise noise - noise on the force sensor readings is $-66 \mathrm{~dB}$ from full scale, allowing $0.1 \mathrm{~N}$ resolution on the tibia axis. The motors are run from the $18 \mathrm{~V}$ supply through PWM H-bridges. Software current control is achieved by correcting for the back EMF, $E=\omega K_{v}$, and dividing by the winding resistance. The electrical time constant of the windings is of the order of the timestep, so it is not significant. An inclinometer will be attached to the spare analogue channels on one of the modules.

The control of biologically-inspired hexapods has often been carried out by distributed processors of minimal complexity. However, a single modern embedded processor now has ample power to control all the legs, and even if the control algorithm is a distributed one it is simpler to implement it as a centralised one. Communication between the legs is purely in software rather than wires. This was the option chosen for Hamlet.

Control software for Hamlet is run by dual Texas Instruments TMS320C44 50MHz 32 bit floating point DSPs on a carrier board that supports up to four modules (Fig. 7). One of them communicates with the serial link by two built-in 32 bit parallel ports. Another parallel port (operated bidirectionally) is used for sending data between the processors for each leg. Functionality is split between the

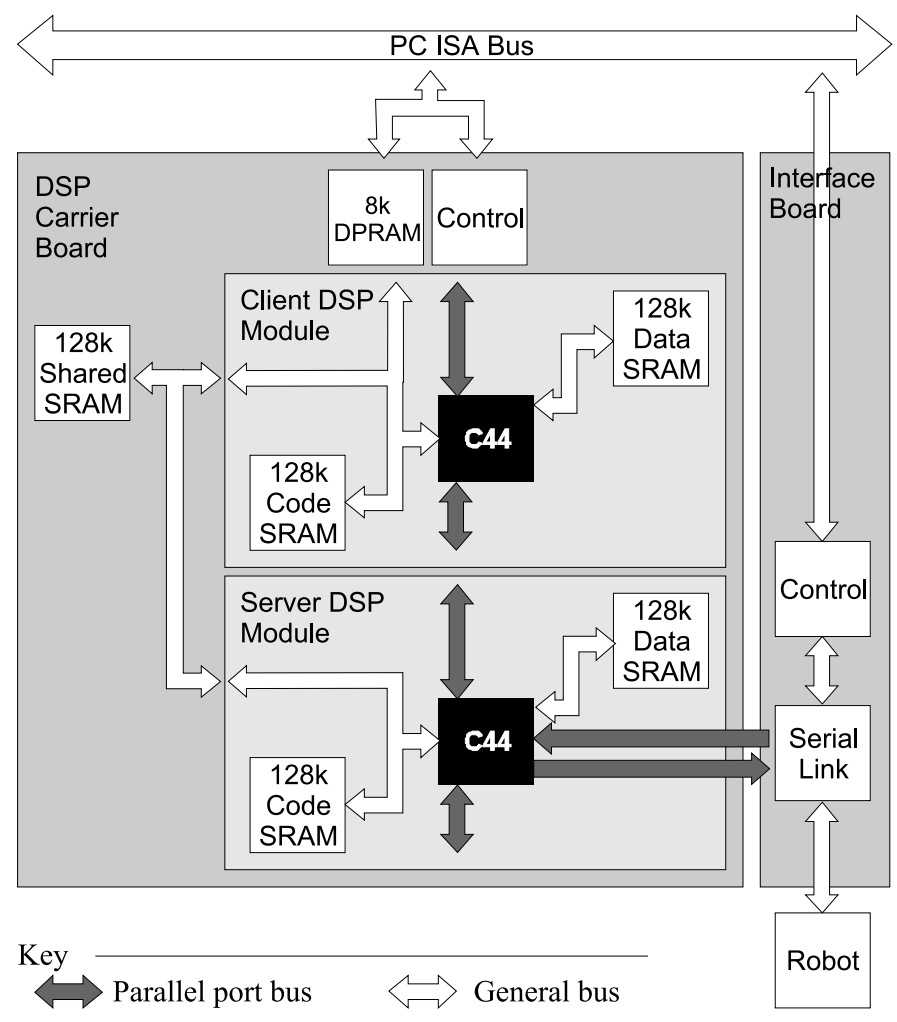

Fig. 7

Two TMS320C44 DSPs ARE used For CONTrolling Hamlet. Boxes labelled CONTROL are for uploading PRograms to the VARIOUS COMPONENTS.

DSPs so that the processor connected to the serial link (server) scales and rotates the raw data to present the current state of each leg to the other processor (client), which runs the control algorithms and returns the desired output torque for each joint. The server analyses data leg by leg and transfers it immediately, allowing the client to run in parallel.

$8 \mathrm{~K}$ words of dual-port memory accessible by the client module are used for interacting with software on the PC. Using GUI software, running on a Pentium II under Windows 95, the user can select operating modes for the DSP code, send commands, inspect and modify variables, plot realtime results and save them to Matlab. Joystick data are continuously sent to the DSPs via the DPRAM. If necessary the client module relays commands to the server using the shared memory.

\section{Force-Position Control}

Many schemes exist for force-position control in rigid manipulators, including Whitney's position accommodation [21], Raibert and Craig's hybrid control [22] and Hogan's impedance control [23]. The method used in Hamlet is position-based compliance control after Lawrence and Stoughton [24] and Kazerooni et al. [25]. Position-based compliance control has the advantages of direct specification of the desired stiffness in task coordinates (in this case 
a gravitational frame centred at the body) and that the position control subsystem can separately take care of the leg dynamics.

Position-based compliance control requires a position control subsystem working in the task coordinate space. In Hamlet this is carried out by simply transforming desired Cartesian positions to joint space.

Joint servo control is by non-linear PID controllers: a deadzone precedes the integral term in order reduce hunting due to the backlash in the drivetrain, and it is also limited to improve gross movement response and prevent damage in the event of a collision.

Compliant control has been implemented on the legs of Hamlet. Software filtering of the raw force measurements is necessary to remove noise and is achieved with a first order low pass Butterworth with corner frequency at $1 \mathrm{~Hz}$. Stiffnesses as low as $500 \mathrm{~N} / \mathrm{m}$ are stable when contacting rocks, despite very low damping in the position controlled subsystem.

\section{Conclusions}

A hexapod walker has been constructed and is virtually ready for experiments. Hardware problems (specifically, angle encoders on five legs were damaged during cleaning) have thus far prevented walking trials from actually being carried out.

Carbon fibre protective boots are presently being constructed to encase the load cells and motors, to protect them from damage when they hit obstacles. These will be equipped with a switch to indicate when a collision has occurred. An inclinometer will also be attached.

Compliant control has been demonstrated, although there is scope for improvement of the stability when contacting hard surfaces by non-linear schemes such as those in Seraji [26]. Damping of the position controlled subsystem is also expected to improve stability under compliant control, while still achieving acceptable performance when not in contact with the environment.

Further research can now be carried out to show that the use of horizontal foot force information to make the feet of a small hexapod walker compliant may improve handling of changes in the environment caused by the robot's walking over it. Global force control may not be necessary to achieve robust, reliable walking, at least for small robots. A 'soft' algorithm, rather than a CPG controlled one, will be used to provide robust gait generation for the robot.

\section{ACKNowledgements}

The authors gratefully acknowledge the efforts of all those involved in the robot's construction, especially Scott Amies, Ken Brown, Andy Cree, Graham Harris, Geoff Leathwick, Julian Murphy and Paul Wells.

\section{REFERENCES}

[1] C. A. Klein and R. L. Briggs, "Use of active compliance in the control of legged vehicles," in IEEE Trans. Systems, Man, and Cybernetics, July 1980, vol. 10, pp. 393-399.
[2] K. J. Waldron, V. J. Vohnout, A. Pery, and R. B. McGhee, "Configuration design of the adaptive suspension vehicle," Journal of Robotics Research, vol. 3, no. 2, pp. 37-48, 1984.

[3] R. A. Brooks, "A robot that walks; emergent behaviors from a carefully evolved network," Neural Computation, vol. 1, pp. 253-262, 1989.

[4] W. Chen, S. H. Yeo, and K. H. Low, "Modular formulation of dynamics of multi-legged robots," in Proc. IEEE International Conference on Robotics and Automation, 1997, pp. 279-284.

[5] F. Pfeiffer, H.-J. Weidemann, and P. Danowski, "Dynamics of the walking stick insect," in Proc. IEEE International Conference on Robotics and Automation, 1990, vol. 3, pp. 1458-1463.

[6] L. Shih, A. A. Frank, and B. Ravani, "Dynamic simulation of legged machines using a compliant joint model," Journal of Robotics Research, vol. 6, no. 4, pp. 33-46, 1987.

[7] J. F. Gardner, "Characteristics and approximations of optimal force distributions in walking machines on rough terrain," in Proc. IEEE International Conference on Advanced Robotics, 1991, pp. 613-618.

[8] M. Schneider and J. Müller, "Force based motion control of the walking machine ALDURO using exact linearization methods," in Proc. IEEE/ASME International Conference on Advanced Intelligent Mechatronics, September 1999, pp. 537-542.

[9] K. J. Waldron, "Force and motion management in legged locomotion," Journal of Robotics and Automation, vol. 2, no. 4, pp. 214-220, December 1986.

[10] H. Weidemann, J. Eltze, and F. Pfeiffer, "Leg design based on biological principles," in Proc. IEEE International Conference on Robotics and Automation, 1993, vol. 3, pp. 352-358.

[11] Ch. Bartling and J. Schmitz, "Reaction to distrubances of a walking leg during stance," Journal of Experimental Biology, vol. 203, pp. 1211-1223, 2000.

[12] E. Celaya and J. M. Porta, "Control of a six-legged robot walking on abrupt terrain," in Proc. IEEE International Conference on Robotics and Automation, 1996, pp. 2731-2736.

[13] K. G. Pearson and R. Franklin, "Characteristics of leg movements and patterns of coordination in locusts walking on rough terrain," Journal of Robotics Research, vol. 3, no. 2, pp. 101$112,1984$.

[14] H. Cruse, "What mechanisms coordinate leg movement in walking arthropods?," Trends in Neuroscience, vol. 13, pp. 15-20, 1990.

[15] H. Cruse, "The control of the anterior extreme position of the hindleg of a walking stick insect, carausius morosus," Physiological Entomology, vol. 4, pp. 121-124, 1979.

[16] J. Dean and G. Wendler, "Stick insect locomotion on a walking wheel: Interleg coordination of leg position," Journal of Experimental Biology, vol. 103, pp. 75-94, 1983

[17] H. Cruse, T. Kindermann, M. Schumm, J. Dean, and J. Schmitz, "Walknet - a biologically inspired network to control six-legged walking," Neural Networks, vol. 11, pp. 1435-1447, 1998.

[18] J. Collins and I. Stewart, "The mathematical springs in insect steps," New Scientist, pp. 36-40, October 1994.

[19] D. Wettergreen and C. Thorpe, "Developing planning and reactive control for a hexapod robot," in Proc. IEEE International Conference on Robotics and Automation, 1996, p. 2718.

[20] E. Krotkov and R. Simmons, "Perception, planning, and control for autonomous walking with the ambler planetary rover," Journal of Robotics Research, vol. 15, no. 2, pp. 155-180, April 1996.

[21] D. E. Whitney, "Force feedback control of manipulator fine motions," Journal of Dynamic Systems, Measurement, and Control, pp. 91-97, 1977.

[22] M. H. Raibert and J. J. Craig, "Hybrid Position/Force control of manipulators," Journal of Dynamic Systems, Measurement and Control, vol. 102, pp. 126-133, 1981.

[23] N. Hogan, "Impedence control: An approach to manipulation: Part i - theory," Journal of Dynamic Systems, Measurement and Control, vol. 107, pp. 1-7, March 1985.

[24] D. A. Lawrence and R. M. Stoughton, "Position-based impedance control: Achieving stability in practice," in Proc. AIAA Guidance, Navigation and Control Conf., 1987, pp. 221226.

[25] H. Kazerooni, T. B. Sheridan, and P. K. Houpt, "Robust compliant motion for manipulators, part i: The fundamental concepts of compliant motion," Journal of Robotics and Automation, vol. 2, no. 2, pp. 83-92, June 1986. 
[26] H. Seraji, "Nonlinear and adaptive control of force and compliance in manipulators," International Journal of Robotics Research, vol. 17, no. 5, pp. 467-484, June 1998. 